differences are matters of degree rather than differences in kind.

This leads directly to the final argument. Rachels proposes that we should be "moral individualists", justifying our ways of treating animals (whether human or nonhuman) on the basis of the characteristics that they have or lack, not on whether or not they belong to a particular group or species. It is thus as unjustifiable to defend "sacrificing" an animal on the grounds that it is not human, as it would be to justify lynching a man on the grounds that he belonged to a particular racial group. If the "sacrifice" is to be defended, then we must point out some relevant difference between the animal subject and human beings. Rachels suggests that the animal's inability to speak is not a morally relevant difference here - for we would not think it appropriate to "sacrifice" human subjects who were mute, or whose linguistic capacities had been destroyed.

Rachels leaves us with some conclusions, and with a challenge. Briefly surveying agricultural practices in the United States, he makes a strong case for the moral wrongness of factory farming methods, and argues that industrial testing of some products brings benefits too ephemeral to outweigh the suffering inflicted on the animals involved. Yet the principal challenge of the book, left unresolved in its final pages, is whether human beings are morally entitled to inflict pain and death on other animals even when there are actual or potential benefits for people. To return to the example with which I began: is it morally coherent to oppose the use of humans as experimental subjects (as, for example, in the Nazi camps), while regarding as justified Banting and Best's experiments on their unfortunate dogs?

There seem to be three lines along which an answer could be sought. One possibility is to scrutinize Rachels' contention that the psychological differences between humans and other animals do not amount to a morally relevant difference in kind. Perhaps our capacity for language and abstract thought brings with it the power to reflect on our own pain and impending death, adding an extra dimension to human suffering. A second approach, remaining within Rachels' framework, would insist that the differences of degree are reflected in the values of human and nonhuman lives. We could respond to the challenge by developing a consequentialist moral calculus. The values of the lives made possible by Banting and Best far outweigh those of the dogs who died prematurely. Finally, appealing to the principle that we can only be morally required to do what is possible for us, we might deny the psychological possibility of refraining from using experiments with animals as a means for alleviating the pain of members of our own species. Could Banting, or anyone in his situation, have watched diabetic children die and resolved to save the dogs instead?

These are only indications of possible lines of response to Rachels' challenging reflections. Created From Animals leaves us with the task of working them out. Forceful, but never strident, it offers a moral perspective which thoughtful people, inside and outside the laboratory, would do well to ponder.

Philip Kitcher is Professor of Philosophy at the Department of Philosophy, 0302, University of California at San Diego, La Jolla, California 92093, USA.

\section{Flies from all angles}

Gerald M. Rubin

Drosophila: A Laboratory Handbook. By Michael Ashburner. Cold Spring Harbor Laboratory Press: 1989. Pp.1,331. \$180. Drosophila: A Laboratory Manual. By Michael Ashburner. Cold Spring Harbor Laboratory Press: 1989. Pp.434. $\$ 50$.

FOR over 80 years the fruitfly Drosophila melanogaster has been an experimental organism of major importance, first for genetics and more recently for cell, developmental and neurobiology. A vast literature of some 40,000 papers has accumulated along with much unpublished folklore. Newcomers to the field several hundred graduate students and postdoctoral fellows each year - not to mention those of us with only a decade or two of experience, are understandably overwhelmed by this body of information and until now have had no practical way to gain access to it. Ashburner has performed an enormous service to the field by distilling nearly all the important facts and methods of Drosophila genetics into an encyclopacdia - Drosophila: A Laboratory Handbook - of some 1,300 pages. This book fills a long recognized void and has been enthusiastically received by the Drosophila research community.

Ashburner provides concise reviews of Drosophila nomenclature, genome structure, developmental biology and evolution in the opening and closing chapters. But the unique and most useful part of the handbook is the central 30 chapters in which the theory and practice of transmission genetics as applied to Drosophila are covered in a clear and comprehensive manner. When one considers the amount and breadth of the material covered over 4,000 different primary sources are cited - it seems remarkable that it took Ashburner only nine years to produce the handbook. It is the very complete and unbiased coverage of the literature, however, that leads to my one mild complaint. Little guidance is given to the reader as to which of the alternative approaches presented should be applied. Consider, for example, the chapter "Mutation and Mutagenesis". Spontaneous mutations as well as those induced by a variety of different methods, including ionizing and ultraviolet radiation, seven different chemical mutagens and viruses are all considered. The metabolism of mutagens, mutator genes, methods for administering mutagenic treatments, breeding schemes and statistical analyses are all discussed. The treatment is impressively comprehensive. If it has ever been done, Ashburner covers it and provides appropriate references for further reading. This extends to methods that most current fly workers are very unlikely to apply, such as vaginal douches to chemically mutagenize sperm. Although the advantages and disadvantages of each mutagen are mentioned in their respective sections, the novice would be greatly aided by a more general discussion of which mutagens and breeding schemes are the most suitable for each of the handful of applications commonly used in current work.

The companion volume Drosophila: $A$ Laboratory Manual contains a collection of 137 protocols covering a wide variety of cytological and molecular techniques used with Drosophila. The protocols are clearly and concisely written and easy to follow. Protocols dealing with genetic methods such as how to administer common chemical mutagens would be a welcome addition. All protocol books, this included, suffer from the fact that active fields evolve rapidly so that many of these protocols will not age well and some are already out of date. Nevertheless, this is a useful volume and the appended lists of reagents and suppliers will be particularly valuable to anyone setting up a new laboratory.

These books are certainly an essential set of references for any laboratory working with Drosophila and I would be surprised if there were many fly labs that did not already own at least one copy. A shorter version of the handbook - say one-third of the length at one third of the price - would find a very wide market among individual students and postdoctoral fellows. This would be especially true if such a textbook of Drosophila genetics focused on that part of the handbook that is truly unique - the chapters on transmission genetics - and emphasized those aspects most relevant to current workers.

Gerald M. Rubin is at the Howard Hughes Medical Institute and the Department of Molecular and Cell Biology, Division of Genetics, University of California, Berkeley, California 94720 , USA. 